\title{
ON THE OSCILLATION OF AN ELLIPTIC EQUATION OF FOURTH ORDER
}

\author{
BHAGAT SINGH
}

Abstract. The elliptic equation

$$
\Delta^{2} u(|x|)+g(|x|) u(|x|)=f(|x|)
$$

is studied for its oscillatory behavior. $\Delta$ is the Laplace operator. Sufficient conditions have been found to ensure that all solutions of this equation continuable in some exterior domain

$$
\Omega=\left\{x=\left(x_{1}, x_{2}, x_{3}\right):|x|>A\right\}
$$

Where

$$
|x|=\left(\sum_{i=1}^{3} x_{i}^{2}\right)^{\frac{1}{2}}
$$

are oscillatory.

\section{Introduction}

Our main purpose in this work is to study the elliptic equation

$$
\Delta^{2} u(|x|)+g(|x|) u(|x|)=f(|x|)
$$

for its oscillatory behavior in a domain $\Omega$ of $R^{3}$ external to the hypersphere

$$
\sum_{i=1}^{3} x_{i}^{2}=A^{2}
$$

Received November 22, 1994; revised April 25, 1995.

1991 Mathematics Subject Classification. Primary 35B05

Key words and phrases. Oscillation, slow oscillation, Laplace, elliptically symmetric. 
where $A>0$ is sufficiently large and $|x|$ is the Euclidian length

$$
|x|=\sqrt{\sum_{i=1}^{3} x_{i}^{2}} .
$$

$g(t)$ and $f(t)$ are continuous functions on $[A, \infty)$ for some $A>0$. In equation (1), $\Delta$ is the Laplace operator so that

$$
\Delta u\left(x_{1}, x_{2}, x_{3}\right)=\sum_{i=1}^{3} \frac{\partial^{2} u}{\partial x_{i}^{2}}
$$

By a solution of (1) we mean a symmetric function $u(|x|)$ which is continuous in some exterior domain

$$
\Omega_{T}=\left\{x=\left(x_{1}, x_{2}, x_{3}\right) \in R^{3}:|x| \geq T\right\}, T \geq A
$$

and satisfies (1).

A function $S(t)$ continuous on $[T, \infty)$ is said to be oscillatory (as in [5], [6]) if $S(t)$ has arbitrarily large zeros in $[T, \infty)$; otherwise $S(t)$ is said to be nonoscillatory. $S(t)$ is said to be slowly oscillating if the set

$$
\left\{\left|t_{m}-t_{n}\right|: S\left(t_{m}\right)=S\left(t_{n}\right)=0, S(t) \neq 0 \text { for } t \in\left(t_{m}, t_{n}\right)\right\}
$$

is unbounded.

We will soon see that equation (1) is closely related to the general ordinary differential equation

$$
L_{n} y(t)+F(t, y(t))=f(t)
$$

which has been, in less general cases, studied in [1-3] and [5-6]. Our techniques and notations in [4-5] will be adapted in this work. Throughout this work we assume that

(i) $L_{0} y(t)=\frac{y(t)}{p_{0}(t)}, L_{i} y(t)=\frac{1}{p_{i}(t)} \frac{d}{d t} L_{i-1} y(t)$ for $1 \leq i \leq n ; \quad n \geq 2$;

(ii) $p_{i}(t), f(t)$ and $g(t)$ are continuous, real valued on $[A, \infty), 1 \leq i \leq n, p_{i}>0$, $i=0,1,2, \cdots, n$; and

$$
\int^{\infty} p_{i}(t) d t=\infty \text { for } \quad 1 \leq i \leq n-1
$$

(iii) $F: R \times R \rightarrow R$ is continuous.

We define as in [5]

$$
\left[\begin{array}{c}
I_{0}=1 \\
I_{k}\left(t, s ; p_{k}, \cdots, p_{1}\right)=\int_{s}^{t} p_{k}(r) I_{k-1}\left(r, s ; p_{k-1}, \cdots, p_{1}\right) d r .
\end{array}\right.
$$


It can be easily verified that

$$
I_{k}\left(t, s ; p_{k}, \cdots, p_{1}\right)=\int_{s}^{t} p_{1}(r) I_{k-1}\left(t, r ; p_{k}, \cdots, p_{2}\right) d r
$$

and

$$
\begin{aligned}
& I_{k}\left(t, s ; p_{k}, \cdots, p_{1}\right)=(-1)^{k} I_{k}\left(s, t ; p_{1}, \cdots, p_{k}\right), \\
& {\left[\begin{array}{c}
J_{k}(t, s)=p_{0}(t) I_{k}\left(t, s ; p_{1}, \cdots, p_{k}\right), \\
K_{k}(t, s)=p_{n}(t) I_{k}\left(t, s ; p_{n-1}, \cdots, p_{n-k}\right) .
\end{array}\right.} \\
& J_{k}(t)=J_{k}(t, A), \quad K_{k}(t)=K_{k}(t, A), \quad 0 \leq K \leq n .
\end{aligned}
$$

It is easy to see that if $p_{i} \equiv 1$ for $0 \leq i \leq n$ then

$$
J_{n}(t)=\frac{(t-A)^{n}}{n !} .
$$

II. Main Results. The following lemma, which is Theorem (1) of Singh and Kusano [5], establishes a growth condition for solutions of equation (5).

Lemma 1. Suppose that (6) holds, and there exists a number $\gamma \in(0,1]$ and a continuous function $q:[A, \infty) \rightarrow[0, \infty)$ such that

$$
|F(t, s)| \leq q(t)|s|^{\gamma} \quad \text { for }(t, s) \in[A, \infty) \times R .
$$

Suppose moreover that

$$
\int^{\infty} p_{n}(t)|f(t)| d t<\infty
$$

and

$$
\int^{\infty}\left[J_{n-1}(t)\right]^{\gamma} p_{n}(t) q(t) d t<\infty .
$$

Then every nontrivial solution $y(t)$ of equation (5) satisfies

$$
y(t)=0\left(J_{n-1}(t)\right) \quad \text { as } t \rightarrow \infty \text {. }
$$

Lemma 2. The function $u(|x|), x=\left(x_{1}, x_{2}, x_{3}\right)$ is a solution of equation (1) in an exterior domain

$$
\Omega=\left\{x \in R^{3}:|x| \geq T\right\}, \quad T \geq A,
$$

if and only if $u(t)$ is a solution of the ordinary differential equation

$$
\frac{1}{t} \frac{d^{4}}{d t^{4}}(t u)+g(t) u=f(t), \quad t \geq T, \quad \text { where } t=|x| .
$$


Proof. By direct substitution, it can be easily verified that

$$
\begin{aligned}
\Delta u(|x|) & =\frac{1}{t^{2}} \frac{d}{d t} t^{2} \frac{d u}{d t} \\
& =\frac{1}{t} \frac{d^{2}}{d t^{2}}(t u), \quad t=|x| \\
\Delta^{2} u(|x|) & =\Delta\left(\frac{1}{t} \frac{d^{2}}{d t^{2}}(t u)\right) \\
& =\frac{1}{t} \frac{d^{2}}{d t^{2}}\left(t \times \frac{1}{t} \frac{d^{2}}{d t^{2}}(t u)\right) \\
& =\frac{1}{t} \frac{d^{2}}{d t^{2}}\left(\frac{d^{2}}{d t^{2}}(t u)\right) \\
& =\frac{1}{t} \frac{d^{2}}{d t^{2}}\left(t \frac{d^{2} u}{d t^{2}}+2 \frac{d u}{d t}\right) \\
& =\frac{1}{t}\left(t \frac{d^{4} u}{d t^{4}}+4 \frac{d^{3} u}{d t^{3}}\right) \\
& =\frac{1}{t} \frac{d^{4}}{d t^{4}}(t u) .
\end{aligned}
$$

By direct substitution from (17) into equation (1), we get equation (16). Conversely if $u(t)$ is a solution of equation (16) where $t=|x|$ then we can obtain equation (1). The proof of Lemma 2 is now complete.

Theorem 1. Suppose

$$
\int^{\infty} t|f(t)| d t<\infty
$$

and

$$
\int^{\infty} t^{3}|g(t)| d t<\infty
$$

Then every symmetric solution $u(|x|)$ of equation (1) satisfies

$$
u(|x|)=0\left(|x|^{2}\right)
$$

as $|x| \rightarrow \infty$.

Proof. If we choose $p_{0}(t)=1 / t, p_{1}(t)=p_{2}(t)=p_{3}(t)=1, p_{4}(t)=t$, and $\gamma=1$, then all conditions of Lemma (1) are satisfied for equation (16). Hence any solution $u(t)$ of this equation satisfies $t u(t)=0\left(t^{3}\right) \Rightarrow u(t)=0\left(t^{2}\right)$. The conclusion now follows by Lemma 2.

Our next lemma is Theorem (3) of our work [5] which gives us a stronger result for equation (1). 
Lemma 3. Suppose that (6) holds and there exists a number $\gamma \in(0,1]$ and a continuous function $q:[A, \infty) \rightarrow[0, \infty)$ such that (13) holds. Suppose moreover that

$$
\int^{\infty} K_{n-1}(t)|f(t)| d t<\infty
$$

and

$$
\int^{\infty}\left[J_{n-1}(t)\right]^{\gamma} K_{n-1}(t) q(t) d t<\infty .
$$

Then every oscillatory solution $y(t)$ of (5) satisfies

$$
\lim _{t \rightarrow \infty}|y(t)| / p_{0}(t)=0 .
$$

This lemma leads us to the following stronger result for equation (1).

Theorem 2. Suppose

$$
\int^{\infty} t^{4}|f(t)| d t<\infty
$$

and

$$
\int^{\infty} t^{6}|g(t)| d t<\infty
$$

Then all oscillatory spherically symmetric solutions $u(|x|)$ of $(1)$ satisfy

$$
\lim _{|x| \rightarrow \infty}|x| u(|x|)=0
$$

Proof. Since

$$
\begin{aligned}
& k_{3}(t)=0\left(t^{3}\right) \\
& J_{3}(t)=0\left(t^{3}\right)
\end{aligned}
$$

and $\gamma=1$, all conditions of Lemma (3) are satisfied for equation (16). Thus any oscillatory solution $u(t)$ of $(16)$ satisfies

$$
\operatorname{Lim}_{t \rightarrow \infty} u(t) / p_{0}(t)=0 .
$$

Since $p_{0}(t)=\frac{1}{t}$, we have $t u(t) \rightarrow 0$ as $t \rightarrow \infty$. The conclusion of the theorem follows.

Our next theorem gives sufficient conditions for all bounded solutions of equation (1) to be oscillatory.

Theorem 3. Suppose that for any $T \geq A$

$$
\operatorname{Lim} \sup _{t \rightarrow \infty}\left(\frac{1}{t^{3}} \int_{T}^{t}(t-s)^{3} s f(s) d s\right)=\infty
$$




$$
\operatorname{Lim} \inf _{t \rightarrow-\infty}\left(\frac{1}{t^{3}} \int_{T}^{t}(t-s)^{3} s f(s) d s\right)=-\infty
$$

and

$$
\operatorname{Lim}_{t \rightarrow \infty} \frac{1}{t^{3}} \int_{T}^{t} s|g(s)|(t-s)^{3} d s<\infty
$$

Then all spherically symmetric bounded solutions $u(|x|)$ of equation (1) defined in an exterior domain

$$
\Omega_{T}=\left\{x=\left(x_{1}, x_{2}, x_{3}\right):|x|>T\right\}
$$

are oscillatory.

Proof. We prove this for equation (16). Let $u(t)$, to the contrary, be a bounded nonoscillatory solution of (16). Without loss of generality suppose that $u(t)>0$ for $t \geq T$. On direct integration from equation (16), we have

$$
\begin{aligned}
& t u^{\prime \prime \prime}(t)-T u^{\prime \prime \prime}(T)-\int_{T}^{t} u^{\prime \prime \prime}(s) d s+4 \int_{T}^{t} u^{\prime \prime \prime}(s) d s \\
& +\int_{T}^{t} s g(s) u(s) d s=\int_{T}^{t} s f(s) d s .
\end{aligned}
$$

On repeated integration three more times, we get

$$
\begin{aligned}
& \frac{t u(t)}{t^{3}}-\frac{M(t)}{t^{3}}+\frac{1}{t^{3}} \int_{T}^{t} \frac{(t-s)^{3}}{3 !} s g(s) u(s) d s \\
= & \frac{1}{t^{3}} \int_{T}^{t} \frac{(t-s)^{3}}{3 !} s f(s) d s
\end{aligned}
$$

where

$$
M(t)=\left(C_{1}+C_{2}(t-T)+C_{3}(t-T)^{2}+C_{4}(t-T)^{3}\right)
$$

and $C_{1}, C_{2}, C_{3}$, and $C_{4}$ are appropriate constants.

Since the left side of (29) is bounded as $t \rightarrow \infty$, and the right hand side swings between $-\infty$ and $\infty$, a contradiction is reached. Hence by virtue of equation (16), all bounded spherically symmetric solutions $u(|x|)$ of equation (1) are oscillatory. This completes the proof.

If in Theorem (3), we require an additional condition that $g(t)>0$ for $t \geq A$, then its proof reveals that boundedness of the solutions of (1) or of (16) is not essential. This leads us to the following stronger theorem.

Theorem 4. In addition to the conditions of Theorem (3), suppose $g(t) \geq 0$ for $t \geq A$. Then all solutions of equation (1) continuable into the exterior domain $\Omega_{T}$ are oscillatory. 
Proof. In reexamination of the proof of Theorem (3), we notice that the only place where boundedness of $u(t)$ is needed is in the integral on the left side of (29). Since $g(t) \geq 0$, it is no longer needed. This observation completes the proof.

Example 1. Consider the elliptic equation

$$
\Delta^{2} u(|x|)+e^{-|x|} u(|x|)=|x|^{5} \sin (|x|)
$$

where

$$
|x|=\left(\sum_{i=1}^{3} x_{i}^{2}\right)^{\frac{1}{2}}
$$

Then the corresponding companion differential equation is

$$
\frac{1}{t} \frac{d^{4}(u t)}{d t^{4}}+\epsilon^{-t} u=t^{5} \sin t, \quad t>0
$$

which satisfies all the conditions of Theorem (3). Hence all solutions of equation(30) continuable beyond the hypersphere

$$
\sum_{i=1}^{3} x_{i}^{2}=T^{2}, \quad T>A .
$$

are oscillatory.

Remark 1. The proof of Theorem (3) reveals that if in addition to (26), (27), $f(t)$ is also slowly oscillating then bounded solutions of equations (16) and (1) will also be slowly oscillating. We state this fact as Theorem (4).

Theorem 5. Suppose that (26), (27) and (28) hold. Further suppose that for any bounded function $L(t)$ the funcition $f(t)-L(t) g(t)$ is slowly oscillating. Then all bounded solutions of equations (16) and (1) are slowly oscillating.

Proof. Let $u(t)$ be a bounded solution of equation (16). Then by Theorem (3), $u(t)$ is oscillatory. The oscillation of $u(t)$ implies oscillation of $u^{I V}(t)$. From equation (16)

$$
\frac{d^{4}}{d t^{4}}(t u(t))=t(f(t)-u(t) g(t))
$$

Since $u(t)$ is bounded, (32) implies that

$$
\frac{d^{4}}{d t^{4}}(t u(t))
$$

is also slowly oscillating. 
We now observe an elementary fact that if the derivative of an oscillatory function $h(t)$ is slowly oscillating so is the function $h(t)$. To see this we note that if $h\left(t_{1}\right)=$ $h\left(t_{2}\right)=0, h(t) \neq 0$ for $t \in\left(t_{1}, t_{2}\right)$ then

$$
\int_{t_{1}}^{t_{2}} h^{\prime}(t) d t=0
$$

Thus between any two consecutive zeros of $h(t)$, there must be a zero of $h^{\prime}(t)$. Hence if $h^{\prime}(t)$ is slowly oscillating, so is $h(t)$. This observation leads to the fact that slow oscillation of

$$
\frac{d^{4}}{d t^{4}}(t u(t))
$$

implies slow oscillation of

$$
\frac{d^{3}}{d t^{3}}(t u(t)), \frac{d^{2}}{d t^{2}}(t u(t)), \frac{d}{d t}(t u(t))
$$

and consequently of $t u(t)$. This completes the proof of Theorem 4 .

Remark 2. Our next example shows that conditions of Theorem (4) are quite practical.

Example 2. Consider the equation

$$
\Delta^{2} u(|x|)+\frac{1}{|x|^{10}} \sin (|x|) u(|x|)=\sin (\log (|x|))
$$

satisfies the conditions and conclusion of this theorem. This follows from the fact that for the companion equation

$$
\frac{1}{t} \frac{d^{4}}{d t^{4}}(t u(t))+\frac{1}{t^{(10)}} \sin (t) u=\sin (\log (t)), \quad t>0
$$

the function

$$
\frac{1}{t^{10}}\left(t^{10} \sin (\log (t))-\sin (t) u(t)\right)
$$

is slowly oscillating for any bounded $u(t)$.

\section{References}

[1] T. Kusano and M. Naito, "Comparison theorems for functional differential equations with deviating arguments," J. Math. Soc. Japan, 33(1981), 509-532.

[2] T. Kusano and M. Naito, "Boundedness of solutions of a class of higher order ordinary differential equations," J. Differential Equations, 46(1982), 32-45.

[3] B. Singh, "General functional differential equations and their asymptotic behavior," The Yokohama Math. J., 24(1976), 125-132. 
[4] B. Singh and T. Kusano, "On asymptotic limits of nonoscillations in functional equations with retarded arguments," Hiroshima Math. J., 10(1980), 557-565.

[5] B. Singh and T. Kusano, "Asymptotic behavior of oscillatory solutions of a differential equation with deviating arguments," J. Math. Anal. Appl., 83(1981), 395-407.

[6] B. Singh, "Minimal existence of nonoscillatory solutions in functional differential equations with deviating arguments," Rocky Mountain J. Math., 14(1984), 531-540.

Bhagat Singh, Department of Mathematics, University of Wisconsin Centers-Manitowoc, 705 Viebahn Street, Manitowoc, WI 54220. U.S.A. 